\title{
Multiple Correlation Analysis of Fuzzy Sets
}

\author{
Nancy P. Lin \\ Department of Computer Science and Information Engineering, Tamkang University, \\ Tamsui, Taipei, Taiwan, R.O.C. \\ nancylin@mail.tku.edu.tw
}

\begin{abstract}
There are so many fuzzy informations in databases waiting to be explored; methods to analyze these fuzzy data are needed. After a series of topics of correlation among fuzzy sets has been studied, namely, simple [3] and partial correlation analysis [4] of fuzzy sets, we want to study more about multiple relationships among fuzzy sets. In this paper, we will generalize the discussion of linear relationship between fuzzy attribute-pairs to linear relationship among one dependent fuzzy attribute and several independent fuzzy attributes, measured by multiple correlation coefficient of fuzzy set. We will also discuss the fact that the regression coefficients of a multiple linear regression model of one dependent fuzzy attribute on several independent fuzzy attributes can be used to obtain the multiple correlation coefficients among the particular dependent and those independent fuzzy attributes in the regression model.
\end{abstract}

\section{Introduction}

Data volumes get larger so rapidly, many data may be fuzzy but useful, waiting to be explored, methods to investigate these fuzzy data are needed. In previous studies [3] and [4], we have discussed the simple and partial correlation analysis on Zadeh's fuzzy sets $[9,10,12,13]$. In analyzing the fuzzy attributes in databases, simple correlation coefficients [3] provides us with a good sense of linear relationship of fuzzy attribute-pairs, while a partial correlation coefficient [4] shows the linear relationship between two fuzzy attributes when the influence of other fuzzy attributes are partialed out.

But, sometimes we have doubts that fuzzy attributes in a database may have contributed to the result of a specific dependent fuzzy attribute. Therefore, in this paper, the idea of correlation between fuzzy attribute-pair will be generalized to finding the linear relationship between one dependent fuzzy attribute and several independent fuzzy attributes, we will measure it by multiple correlation coefficients of fuzzy attributes.

In Section 2, a definition of multiple correlation coefficients of fuzzy attributes is given and properties of multiple correlation coefficients are noted. The same fuzzy data set from the university student activity example in [4] are used in computing the multiple correlation coefficients.

Relationship between multiple linear regression and multiple correlation coefficient are discussed in Section 3. A statistics, coefficient of multiple determinations, can be 
obtained from the regression coefficients of a multiple regression model, which measures the combined effect of independent fuzzy attributes on the dependent fuzzy attribute in the regression model. In fact, this coefficient of multiple determinations is the square of multiple correlation coefficient, we have a study on this matter.

In the last paragraph, we will talk about the related area of further studies. Methods proposed in this paper and papers [3] and [4], may be applied to discover characteristics and the relationships among the fuzzy data in order to generate useful rules in data mining of fuzzy data.

\section{Multiple Correlation Analysis of Fuzzy Sets}

Here, multiple correlation coefficients are defined for considering $\mathrm{p}$ independent fuzzy attributes $\boldsymbol{A}_{1}, \boldsymbol{A}_{2}, \cdots, \boldsymbol{A}_{p}$ to correlate one dependent fuzzy attribute $\boldsymbol{O}$.

Suppose we have a random sample $\left(\boldsymbol{x}_{1}, \boldsymbol{x}_{2} ; \cdots, \boldsymbol{x}_{\mathrm{n}}\right)$, drawn from a crisp set $\mathbf{X}$. Membership grades, $\left\{\mu_{\boldsymbol{O}}\left(\boldsymbol{x}_{\mathrm{i}}\right)\right.$ and $\mu_{\boldsymbol{A j}}\left(\boldsymbol{x}_{\mathrm{i}}\right)$, for $\left.\mathrm{j}=1, \ldots, p\right\}$, of dependent fuzzy attribute $\boldsymbol{O}$ and independent fuzzy attributes $\boldsymbol{A}_{1}, \boldsymbol{A}_{2}, \cdots, \boldsymbol{A}_{p}$ are attached to each observation $\boldsymbol{x}_{\mathrm{i}}$. Now we want to examine the linear relationship between dependent fuzzy attribute $\boldsymbol{O}$ and independent fuzzy attributes $\boldsymbol{A}_{1}, \boldsymbol{A}_{2}, \cdots, \boldsymbol{A}_{p}$ by multiple correlation coefficient, $\mathrm{R}_{\boldsymbol{O} \bullet 1,2, \cdots, p}$, defined as follows:

Definitation 1. Multiple correlation coefficients for fuzzy sets (i). In order to find the multiple correlation between a dependent attribute $\boldsymbol{O}$ and some independent fuzzy attributes $\boldsymbol{A}_{1}, \boldsymbol{A}_{2}, \cdots, \boldsymbol{A}_{p}$, the sample covariance matrix needs to be computed first as in [4], it is then partitioned into four parts $\boldsymbol{S}=\left[\begin{array}{ll}\boldsymbol{S}_{11} & \boldsymbol{S}_{12} \\ \boldsymbol{S}_{21} & \boldsymbol{S}_{22}\end{array}\right]$ where $\boldsymbol{S}_{11}=S_{\boldsymbol{O O}}=\boldsymbol{S}_{\boldsymbol{O}}^{2}$ is the sample variance of fuzzy attribute $\boldsymbol{O} . \boldsymbol{S}_{12}$ is the transpose of $S_{21}$, and represents the vector of covariance, $S_{O_{j}}$, of fuzzy attribute-pair $\left(\boldsymbol{A}_{\boldsymbol{O}}, \boldsymbol{A}_{\mathrm{j}}\right)$, where $\mathrm{j}=1, \ldots, p . \quad \boldsymbol{S}_{22}$ is a symmetric $p \times p$ matrix, represents the covariance $s_{i j}$ of fuzzy attribute-pair $\left(\boldsymbol{A}_{i}, \boldsymbol{A}_{\mathrm{j}}\right)$, where $\mathrm{i}=1, \ldots p$ and $\mathrm{j}=1, \ldots, p$.

The multiple correlation coefficient between fuzzy attribute $\boldsymbol{O}$ and fuzzy attributes $\boldsymbol{A}_{1}, \boldsymbol{A}_{2}, \cdots, \boldsymbol{A}_{p}$ can then be obtained by

$$
\mathrm{R}_{\boldsymbol{\sigma} \bullet, \cdots, p}=\sqrt{\frac{\boldsymbol{S}_{12} \boldsymbol{S}_{22}^{-1} \boldsymbol{S}_{21}}{\boldsymbol{S}_{11}}}
$$

Let us illustrate this result in a simple case when $p=2$. Assume that we have three fuzzy attributes $\boldsymbol{O}, \boldsymbol{A}_{1}$ and $\boldsymbol{A}_{2}$, we are interested in finding the multiple correlation 
coefficient of dependent fuzzy attribute $\boldsymbol{O}$ on independent fuzzy attributes $\boldsymbol{A}_{1}$ and $\boldsymbol{A}_{2}$. The first thing is to compute the sample covariance matrix

$$
\boldsymbol{S}=\left[\begin{array}{ccc}
s_{\boldsymbol{O O}} & s_{1 O} & s_{2 \boldsymbol{O}} \\
s_{\boldsymbol{O} 1} & s_{11} & s_{12} \\
S_{\boldsymbol{O} 2} & s_{21} & s_{22}
\end{array}\right]
$$

which can be partitioned into four parts, that is:

$$
\left.\boldsymbol{S}=\left[\begin{array}{ll}
\boldsymbol{S}_{11} & \boldsymbol{S}_{12} \\
\boldsymbol{S}_{21} & \boldsymbol{S}_{22}
\end{array}\right]=\left[\begin{array}{l}
{\left[s_{\boldsymbol{O O}}\right.}
\end{array}\right]\left[\begin{array}{ll}
{\left[\begin{array}{ll}
s_{1 \boldsymbol{O}} & \boldsymbol{S}_{2 \boldsymbol{O}}
\end{array}\right]} \\
s_{\boldsymbol{O} 1} \\
s_{\boldsymbol{O} 2}
\end{array}\right]\left[\begin{array}{ll}
s_{11} & s_{12} \\
s_{21} & s_{22}
\end{array}\right]\right]
$$

Where $\boldsymbol{S}_{11}$ is the sample variance of fuzzy attribute $\boldsymbol{O}$. $\boldsymbol{S}_{12}$, a $2 \times 1$ vector and the transpose of $\boldsymbol{S}_{21}$, represents the covariance-vector of fuzzy attribute $\boldsymbol{O}$ and fuzzy attributes $\boldsymbol{A}_{1}$ and $\boldsymbol{A}_{2} \cdot \boldsymbol{S}_{22}$ is the symmetric covariance-matrix of fuzzy attributes $\boldsymbol{A}_{1}$ and $\boldsymbol{A}_{2}$. Then by using equation (2.1), multiple correlation coefficient of fuzzy attribute $\boldsymbol{O}$ on fuzzy attributes $\boldsymbol{A}_{1}$ and $\boldsymbol{A}_{2}$ can be computed

$$
\begin{aligned}
& \mathrm{R}_{\boldsymbol{O} \bullet 12}=\left[\frac{\left[\begin{array}{ll}
s_{1 O} & s_{2 O}
\end{array}\right] \times\left[\begin{array}{ll}
s_{11} & s_{12} \\
s_{21} & s_{22}
\end{array}\right]^{-1} \times\left[\begin{array}{l}
s_{\boldsymbol{O} 1} \\
s_{\boldsymbol{O} 2}
\end{array}\right]}{s_{\boldsymbol{O O}}}\right]^{1 / 2} \\
& =\left[\frac{\left[\begin{array}{ll}
s_{1 O} & s_{2 O}
\end{array}\right] \times \frac{1}{\left(s_{11} s_{22}-s_{12} s_{21}\right)}\left[\begin{array}{cc}
s_{22} & -s_{12} \\
-s_{21} & s_{11}
\end{array}\right] \times\left[\begin{array}{l}
s_{\boldsymbol{O} 1} \\
s_{\boldsymbol{O} 2}
\end{array}\right]}{s_{\boldsymbol{O O}}}\right]^{1 / 2} \\
& =\left[\frac{\left[s_{1 O} s_{22}-s_{2 O} s_{21}-s_{1 O} s_{12}+s_{2 O} s_{11}\right] \times\left[\begin{array}{c}
s_{\boldsymbol{O} 1} \\
s_{\boldsymbol{O} 2}
\end{array}\right]}{\left(s_{11} s_{22}-s_{12} s_{21}\right) \times s_{\boldsymbol{O O}}}\right]^{1 / 2} \\
& =\left[\frac{\left(s_{22} s_{\boldsymbol{O} 1}-s_{\boldsymbol{O} 2} s_{12}\right) s_{\boldsymbol{O} 1}+\left(s_{11} s_{\boldsymbol{O} 2}-s_{\boldsymbol{O} 1} s_{12}\right) s_{\boldsymbol{O} 2}}{\left(s_{11} s_{22}-s_{12}^{2}\right) \times s_{\boldsymbol{O O}}}\right]^{1 / 2}
\end{aligned}
$$




$$
=\left[\frac{\left(s_{22} s_{\boldsymbol{O} 1}-s_{\boldsymbol{O} 2} s_{12}\right)}{\left(s_{11} s_{22}-s_{12}^{2}\right)} \cdot \frac{s_{\boldsymbol{O} 1}}{s_{\boldsymbol{O O}}}+\frac{\left(s_{11} s_{\boldsymbol{O} 2}-s_{\boldsymbol{O} 1} s_{12}\right)}{\left(s_{11} s_{22}-s_{12}^{2}\right)} \cdot \frac{s_{\boldsymbol{O} 2}}{s_{\boldsymbol{O O}}}\right]^{1 / 2} .
$$

Property 1. Properties of Multiple correlation coefficients. In the case of $p=2$, for any two independent fuzzy attributes $\boldsymbol{A}_{1}$ and $\boldsymbol{A}_{2}$, the properties that should be satisfied by multiple correlation coefficient $\mathrm{R}_{\boldsymbol{O} \bullet 1,2}$ are:

(i) $\mathrm{R}_{o \bullet 1,2}=\mathrm{R}_{o \bullet 2,1}$.

(ii) $\mathrm{R}_{\boldsymbol{O} \bullet 1,2}$ reaches its minimum when $r_{12}=0$, where $r_{12}$ is the simple correlation coefficient between fuzzy attributes $\boldsymbol{A}_{1}$ and $\boldsymbol{A}_{2}$.

$$
\text { Since } \begin{aligned}
\mathrm{R}_{O \bullet 1,2} & =\left[\frac{\left[\begin{array}{ll}
s_{1 O} & s_{2 O}
\end{array}\right] \times\left[\begin{array}{ll}
s_{11} & s_{12} \\
s_{21} & s_{22}
\end{array}\right]^{-1} \times\left[\begin{array}{l}
s_{O 1} \\
s_{O 2}
\end{array}\right]}{s_{O O}}\right]^{1 / 2} \\
& =\left[\frac{\left(s_{22} s_{O 1}-s_{O 2} s_{12}\right) s_{O 1}+\left(s_{11} s_{O 2}-s_{O 1} s_{12}\right) s_{O 2}}{\left(s_{11} s_{22}-s_{12}^{2}\right) \times s_{O O}}\right]^{1 / 2}
\end{aligned}
$$

Divide both the numerator and the denominator by $s_{O o} S_{11} s_{22}$, we have

$$
\begin{aligned}
\mathrm{R}_{O \bullet 1,2} & =\left[\frac{\frac{s_{O 1}^{2}}{s_{O O} s_{11}}-\frac{s_{O 1} s_{O 2} s_{12}}{s_{O O} s_{11} s_{22}}+\frac{s_{O 2}^{2}}{s_{O O} s_{22}}-\frac{s_{O 1} s_{O 2} s_{12}}{s_{O O} s_{11} s_{22}}}{1-\frac{s_{12}^{2}}{s_{11} s_{22}}}\right]^{1 / 2} \\
& =\left[\frac{r_{O 1}^{2}-2 \frac{s_{O 1} s_{O 2} s_{12}}{s_{O O} s_{11} s_{22}}+r_{O 2}^{2}}{1-r_{12}^{2}}\right]^{1 / 2}
\end{aligned}
$$

(iii) $\mathrm{R}_{\boldsymbol{O} \bullet 1,2}$ is not defined, if $r_{12}=1$, we can see that from the last equation. 
(iv) $0 \leq \mathrm{R}_{\boldsymbol{O} \bullet 1,2} \leq 1$. Since $\mathrm{R}_{\boldsymbol{O} \bullet 1,2}^{2}$ is a ratio of partial variance to total variance, which is positive and always $\leq 1$, therefore, the positive square root of it is $0 \leq \mathrm{R}_{o \bullet A, C} \leq 1$.

An example, in previous discussion of partial correlation analysis [4], we have used a university student activity sample and noticed that fuzzy attributes $\boldsymbol{A}, \boldsymbol{C}$, and $\boldsymbol{E}$ may have contributed to the result of fuzzy attribute $\boldsymbol{O}$. We refer the fuzzy attribute $\boldsymbol{O}$, the objective accomplishment, as a dependent attribute, and fuzzy attributes $\boldsymbol{A}, \boldsymbol{C}$, and $\boldsymbol{E}$ as independent attributes, representing group atmosphere, group leader, and group environment respectively. The result of multiple correlation analysis ought to show how the independent fuzzy attributes $\boldsymbol{A}, \boldsymbol{C}$, and $\boldsymbol{E}$ may be involved to what extent in the result of the dependent fuzzy attribute $\boldsymbol{O}$ in this fuzzy data set.

Multiple correlation coefficients between dependent fuzzy attribute $\boldsymbol{O}$ and pairs of combinations of independent fuzzy attributes $\boldsymbol{A}, \boldsymbol{C}$, and $\boldsymbol{E}$ can be computed from equation (2.1): $\mathrm{R}_{\boldsymbol{O} \bullet A C}=0.4998, \mathrm{R}_{\boldsymbol{O} \bullet A E}=0.4850, \mathrm{R}_{O \bullet C E}=0.2144$.

Similarly, multiple correlation coefficient of the dependent fuzzy attribute $\boldsymbol{O}$ regarding to three independent fuzzy attributes $\boldsymbol{A}, \boldsymbol{C}$, and $\boldsymbol{E}$, can be computed:

$\mathrm{R}_{O \bullet A C E}=0.5024$.

\section{Multiple Linear Regression vs. Multiple Correlation Coefficient of Fuzzy Sets}

In a database including an interesting dependent fuzzy attribute $\boldsymbol{O}$ and some related independent fuzzy attributes $\boldsymbol{A}_{1}, \boldsymbol{A}_{2}$ and $\boldsymbol{A}_{3}$, attached with their membership grades, $\left\{\mu_{\boldsymbol{O}}\left(\boldsymbol{x}_{\mathrm{i}}\right)\right.$ and $\left.\mu_{A_{1}}\left(\boldsymbol{x}_{\mathrm{i}}\right), \mu_{A_{2}}\left(\boldsymbol{x}_{\mathrm{i}}\right), \mu_{A_{3}}\left(\boldsymbol{x}_{\mathrm{i}}\right)\right\}$, now, let us consider a simple fuzzy linear regression line of fuzzy attribute $\boldsymbol{O}$ on fuzzy attribute $\boldsymbol{A}_{1}$, $\hat{\mu}_{o}=b_{0}+b_{1} \mu_{A 1},\left(\mu_{O}\right.$ and $\mu_{A 1}$ are as defined in previous section $)$. The effect of independent fuzzy attribute $\boldsymbol{A}_{1}$ on the variance of $\boldsymbol{O}$, can be measured by a coefficient of simple determination $r_{\boldsymbol{O} 1}^{2}$. This means that independent fuzzy attribute $\boldsymbol{A}_{1}$ accounts for $100\left(r_{\boldsymbol{O} 1}^{2}\right) \%$ of the total variance of dependent fuzzy attribute $\boldsymbol{O}$. The coefficient of simple determination can be found from the regression coefficient,

$$
\mathrm{r}_{O 1}^{2}=\left[\frac{s_{O 1}}{s_{O} s_{1}}\right]^{2}=\frac{s_{O 1}}{s_{11}} \cdot \frac{s_{O 1}}{s_{O O}}=\boldsymbol{b}_{1} \cdot \frac{s_{O 1}}{s_{O O}}=\mathrm{R}_{O \bullet 1}^{2} \quad \because \boldsymbol{b}_{1}=\frac{s_{O 1}}{s_{11}} .
$$

Here, $s_{O O}, s_{O 1}, s_{11}$ are the same as in equation (2.2).

Where $\mathrm{R}_{\boldsymbol{O} \bullet 1}$ is the multiple correlation coefficient, as from previous section, of dependent fuzzy attribute $\boldsymbol{O}$ with only one independent fuzzy attribute $\boldsymbol{A}_{1}$. And in this 
special case of only one independent fuzzy attribute, $\mathrm{R}_{\boldsymbol{O} \bullet 1}$ is actually the simple correlation coefficient [3], $\boldsymbol{r}_{\boldsymbol{O} 1}$, between $\boldsymbol{O}$ and $\boldsymbol{A}_{1}$.

Next, let us examine the combined effect of independent fuzzy attributes $\boldsymbol{A}_{1}$ and $\boldsymbol{A}_{2}$ on the dependent fuzzy attribute $\boldsymbol{O}$ in the fuzzy linear regression model, $\hat{\mu}_{\boldsymbol{o}}=\boldsymbol{b}_{0}+\boldsymbol{b}_{1} \mu_{A 1}+\boldsymbol{b}_{2} \mu_{A 2}$. This effect can be measured by the coefficient of multiple determination, $\mathrm{R}_{\boldsymbol{O} \bullet 12}^{2}$, that means that $100\left(\mathrm{R}_{\boldsymbol{O} \bullet 12}^{2}\right) \%$ of the total variance of dependent fuzzy attribute $\boldsymbol{O}$ is accounted for by independent fuzzy attributes $\boldsymbol{A}_{1}$ and $\boldsymbol{A}_{2}$. Where $\mathrm{R}_{\boldsymbol{O} \bullet 12}$ is the multiple correlation coefficient of $\boldsymbol{A}_{1}$ and $\boldsymbol{A}_{2}$ on $\boldsymbol{O}$.

The coefficient of multiple determinations can also be obtained from the regression coefficients of multiple regression line by

$$
\mathrm{R}_{\boldsymbol{O} \bullet 12}^{2}=\boldsymbol{b}_{1} \cdot \frac{s_{\boldsymbol{O} 1}}{s_{\boldsymbol{O O}}}+\boldsymbol{b}_{2} \cdot \frac{s_{\boldsymbol{O} 2}}{s_{\boldsymbol{O O}}} .
$$

Here, $s_{O O}, s_{O 1}, S_{O 2}$ are the same as in equation (2.2).

Now, a third independent fuzzy attribute $\boldsymbol{A}_{3}$ is introduced into the regression model, $\hat{\mu}_{\boldsymbol{O}}=\boldsymbol{b}_{0}+\boldsymbol{b}_{1} \mu_{\boldsymbol{A} 1}+\boldsymbol{b}_{2} \mu_{\boldsymbol{A} 2}+\boldsymbol{b}_{3} \mu_{A 3}$, the effect of combining all three independent fuzzy attributes, $\boldsymbol{A}_{1}, \boldsymbol{A}_{2}$ and $\boldsymbol{A}_{3}$ on the dependent fuzzy attribute $\boldsymbol{O}$, is measured by the coefficient of multiple determination, $\mathrm{R}_{\boldsymbol{O} \bullet 123}^{2}$, from which we know that $100\left(\mathrm{R}_{\boldsymbol{O} \cdot 123}^{2}\right) \%$ of the total variance in dependent fuzzy attribute $\boldsymbol{O}$ is accounted for by attributes $\boldsymbol{A}_{1}, \boldsymbol{A}_{2}$ and $\boldsymbol{A}_{3}$.

Also, the coefficient of multiple determination can be computed from the regression coefficients as:

$$
\mathrm{R}_{\boldsymbol{O} \cdot 123}^{2}=\boldsymbol{b}_{1} \cdot \frac{s_{\boldsymbol{O} 1}}{s_{\boldsymbol{O O}}}+\boldsymbol{b}_{2} \cdot \frac{s_{\boldsymbol{O} 2}}{s_{\boldsymbol{O O}}}+\boldsymbol{b}_{3} \cdot \frac{s_{\boldsymbol{O} 3}}{s_{\boldsymbol{O O}}} .
$$

Here, $s_{O O}, S_{O 1}, S_{O 2}, s_{O 3}$ are the same as in equation (2.2), and $\mathrm{R}_{O \bullet 123}$ is the multiple correlation coefficient of $\boldsymbol{A}_{1}, \boldsymbol{A}_{2}$ and $\boldsymbol{A}_{3}$ on $\boldsymbol{O}$. From the discussion of this section, we have another way of determine multiple correlation coefficients.

Definitation 2. Multiple correlation coefficients for fuzzy sets (ii). If the multiple linear regression equation of independent fuzzy attributes $\boldsymbol{A}_{1}, \boldsymbol{A}_{2}, \cdots, \boldsymbol{A}_{p}$ on the dependent fuzzy attribute $\boldsymbol{O}$ is known,

$$
\hat{\mu_{o}}=b_{0}+b_{1} \mu_{A 1}+b_{2} \mu_{A 2}+\cdots+b_{p} \mu_{A p} .
$$


Then the multiple correlation coefficient between fuzzy attribute $\mathrm{O}$ and independent fuzzy attributes $\boldsymbol{A}_{1}, \boldsymbol{A}_{2}, \cdots, \boldsymbol{A}_{p}$ can be found by the formula:

$$
\mathrm{R}_{\boldsymbol{O} \bullet 1,2, \cdots, p}=\sqrt{\frac{\sum_{\mathrm{i}=1}^{p} \boldsymbol{b}_{\mathrm{i}} s_{\boldsymbol{O}}}{s_{\boldsymbol{O O}}}} .
$$

Where $s_{O O}$ and $s_{O i}, i=1, \cdots, p$ are the same as in equation (2.2).

An example, from the result of the university student activity sample [3,4], we obtain the simple linear regression line of dependent fuzzy attribute $\boldsymbol{O}$ on independent fuzzy attributes $\boldsymbol{A}, \hat{\mu}_{o}=0.3542+0.5771 \mu_{A}$. Then, coefficient of simple determination can be obtained by equation (3.2):

$$
r_{\boldsymbol{O A}}^{2}=0.2352=(0.4850)^{2}=\mathrm{R}_{\boldsymbol{O} \bullet \boldsymbol{A}}^{2} .
$$

Which means, $23.52 \%$ of the total variation of dependent fuzzy attribute $\boldsymbol{O}$ (degree of the objective accomplishment), is explained by fuzzy attribute $\boldsymbol{A}$ (group atmosphere). Or, the simple correlation coefficient between fuzzy attribute $\boldsymbol{O}$ and fuzzy attribute $\boldsymbol{A}$ is 0.4850 , as that in [3].

When two independent fuzzy attributes $\boldsymbol{A}, \boldsymbol{C}$ are introduced into the regression model, we have $\hat{\mu}_{o}=0.1514+0.5500 \mu_{A}+0.2420 \mu_{C}$. The coefficient of multiple determination can be obtained from equation (3.1):

$$
\begin{aligned}
\mathrm{R}_{\boldsymbol{O} \bullet A C}^{2} & =b_{1} \cdot \frac{s_{\boldsymbol{O A}}}{s_{\boldsymbol{O O}}}+\boldsymbol{b}_{2} \cdot \frac{s_{\boldsymbol{O C}}}{s_{\boldsymbol{O O}}} \\
& =0.5500 \cdot \frac{0.005}{0.012}+0.2420 \cdot \frac{0.001}{0.012} \\
& =0.2498=(0.4998)^{2}
\end{aligned}
$$

That means, $24.98 \%$ of the total variance of dependent fuzzy attribute $\boldsymbol{O}$ is explained by the combined effect of $\boldsymbol{A}$ (group atmosphere) and $\boldsymbol{C}$ (characteristics of group leader). It also leads to that the multiple correlation coefficient of $\boldsymbol{A}$ and $\boldsymbol{C}$ on $\boldsymbol{O}$ is 0.4998 , which corresponds to the result in previous paragraph.

Now, independent fuzzy attribute $\boldsymbol{E}$ (group environment) is added to the regression model, we have $\hat{\mu}_{o}=0.1415+0.5638 \mu_{A}+0.2918 \mu_{C}-0.0579 \mu_{E}$. Then the coefficient of multiple determination computed from equation (3.1)

$$
\begin{aligned}
\mathrm{R}_{\boldsymbol{O} \bullet A C E}^{2} & =b_{1} \cdot \frac{s_{\boldsymbol{O A}}}{s_{\boldsymbol{O O}}}+\boldsymbol{b}_{2} \cdot \frac{s_{\boldsymbol{O C}}}{s_{\boldsymbol{O O}}}+\boldsymbol{b}_{3} \cdot \frac{s_{\boldsymbol{O E}}}{s_{\boldsymbol{O O}}} \\
& =0.5638 \cdot \frac{0.005}{0.012}+0.2918 \cdot \frac{0.001}{0.012}-0.0579 \cdot \frac{0.002}{0.012}
\end{aligned}
$$




$$
\begin{aligned}
& =0.2524 \\
& =(0.5024)^{2} .
\end{aligned}
$$

This figure shows that $25.24 \%$ of the total variance of dependent fuzzy attribute $\boldsymbol{O}$ is explained by the combined effect of all three independent fuzzy attributes $\boldsymbol{A}, \boldsymbol{C}$ and $\boldsymbol{E}$. Also, we can see that the multiple correlation coefficient of $\boldsymbol{A}, \boldsymbol{C}$ and $\boldsymbol{E}$ on dependent fuzzy attribute $\boldsymbol{O}$ is 0.5024 as obtained from equation (2.1) in previous section.

\section{Conclusions}

Correlation analysis among attributes in a database usually interests us; the correlation analysis for ordinary crisp data has been discussed for a long time in conventional statistics and related applications [1,2,5]. In the mean time, many fuzzy attributes existed in databases are getting more and more interested to the researchers; our objective is to extend the correlation analysis to fuzzy attributes in analyzing fuzzy data. By adopting the methods from mathematical statistics, definitions has been proposed for simple correlation coefficient on fuzzy sets [3], partial correlation coefficient on fuzzy sets [4], and, in this paper, multiple correlation coefficient on fuzzy sets.

As data volumes grow exponentially, massive fuzzy information in databases have not yet been discovered, which may represent important facts or past experiences. To mine knowledge and generate useful rules from fuzzy data is our goal to pursue. Methods proposed in this paper and papers [3] and [4], can be applied to discover characteristics and the relationships among the fuzzy data in order to generate useful rules in data mining of fuzzy data.

\section{References}

1. T. W. Anderson: An Introduction to Multivariate Statistical Analysis, 2nd Ed. John Wiley \& Sons, 1984.

2. S. F. Arnold: Mathematical Statistics, Prentice-Hall, New Jersey, 1990.

3. D. A. Chiang and N. P. Lin: Correlation of Fuzzy Sets, Fuzzy Sets and Systems, Vol. 102 (1999) 221-226.

4. D. A. Chiang and N. P. Lin: Partial Correlation of Fuzzy Sets, Fuzzy Sets and Systems, Vol. 110 (2000) 209-215.

5. S. Dowdy and S. Wearden: Statistics for Research, John Wiley \& Sons, 1983.

6. G. J. Klir and T. A. Folger: Fuzzy Sets, Uncertainty, and Information, Prentice-Hall International, Inc. 1988.

7. G. J. Klir and B. Yuan: Fuzzy Sets and Fuzzy Logic, Theory and Applications, Prentice-Hall International, Inc. 1995.

8. L. A. Zadeh: Fuzzy sets, Information and Control, Vol. 8 (1965) 338-353.

9. H.-J. Zimmermann: Fuzzy Set Theory and Its Applications, 2nd Ed., Kluwer Academic Publishers, 1991. 